\title{
Impact of the therapeutic positioning report in the P\&R process in Spain: analysis of orphan drugs approved by the European Commission and reimbursed in Spain from 2003 to 2019
}

Xavier Badia ${ }^{1 *}\left(\mathbb{D}\right.$, Tania Vico ${ }^{1}$, John Shepherd ${ }^{1}$, Alicia Gil ${ }^{1}$, José Luis Poveda-Andrés ${ }^{2}$ and César Hernández ${ }^{3}$

\begin{abstract}
Background: Pricing and reimbursement decisions for orphan drugs are faced with differences access between European countries depending on each reimbursement policies, evaluation processes and timings. In 2013, the therapeutic positioning report was introduced in the pricing and reimbursement process in Spain. The present study aims to identify orphan drugs authorised in Spain and approved by the European Commission between January 2003 and December 2019, analyse the impact of the therapeutic positioning report in the pricing and reimbursement process of orphan drugs in Spain and to assess additional potential criteria that could influence pricing and reimbursement decisions for orphan drugs.
\end{abstract}

Results: Ninety-four orphan drugs have been approved by the European Commission between January 2003 and December 2019 and have marketing authorisation in Spain. Out of the 94 orphan drugs, 46 (48.9\%) had received pricing and reimbursement approval. Before the inclusion of the therapeutic positioning report in year 2013, the mean time from European Commission approval to pricing and reimbursement approval for orphan drugs in Spain was $25.1 \pm 16.5$. After 2013, timelines have been reduced by an average of 9 months. The mean regulatory time from European Commission approval to Spanish marketing authorisation has decreased nearly 4 months (from $7.5 \pm 10.2$ months in years 2003-2013 to $3.8 \pm 7.6$ months in years 2014-2019). The instauration of the therapeutic positioning report could be associated with a reduction of the mean time from the Spanish marketing authorisation to pricing and reimbursement approval by an average of 5 months (from $17.3 \pm 13.1$ months in years 2003-2013 to $12.3 \pm 5$ months in years 2014-2019). In addition, orphan drugs with a positive conclusion in the therapeutic positioning report would be more likely to be reimbursed in Spain $(p<0,0001)$.

Conclusions: This study shows that the therapeutic positioning report plays a key role in the pricing and reimbursement process in Spain. A positive conclusion of the therapeutic positioning report seems to favourably affect pricing and reimbursement decisions in Spain and, since its introduction, has also contributed to reduce pricing and reimbursement approval timelines in Spain.

Keywords: Orphan drugs, Pricing, Reimbursement, Spain

\footnotetext{
* Correspondence: xbadia@omakaseconsulting.com

'Omakase Consulting S.L., Barcelona, Spain

Full list of author information is available at the end of the article
}

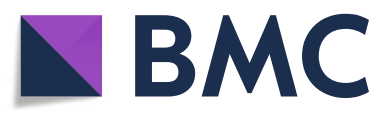

(c) The Author(s). 2020 Open Access This article is licensed under a Creative Commons Attribution 4.0 International License, which permits use, sharing, adaptation, distribution and reproduction in any medium or format, as long as you give appropriate credit to the original author(s) and the source, provide a link to the Creative Commons licence, and indicate if changes were made. The images or other third party material in this article are included in the article's Creative Commons licence, unless indicated otherwise in a credit line to the material. If material is not included in the article's Creative Commons licence and your intended use is not permitted by statutory regulation or exceeds the permitted use, you will need to obtain permission directly from the copyright holder. To view a copy of this licence, visit http://creativecommons.org/licenses/by/4.0/ The Creative Commons Public Domain Dedication waiver (http://creativecommons.org/publicdomain/zero/1.0/) applies to the data made available in this article, unless otherwise stated in a credit line to the data. 


\section{Background}

Health Technology Assessment (HTA) country appraisals of Orphan Drugs (ODs) face added challenges due to the intrinsic characteristics of rare diseases from a clinical and economic point of view. The limited knowledge and heterogeneity of the diseases, the limitations in clinical trial development due to small and typically heterogeneous patient populations and the use of indirect endpoints [1] make it difficult to demonstrate added clinical benefit [2, 3].

While orphan designation and marketing authorisation occurs at European level, access to ODs remains a member state responsibility [4], resulting in differences and restrictions in access for patients due to differences in national reimbursement policies, processes and timings $[5,6]$.

Rare Diseases are a concerning health problem in Spain as they affect altogether about 3 million of patients (6,5\% of the Spanish population) [7]. Reimbursement criteria for innovative medicines (including ODs) are explicitly defined in the Spanish legislation [8]. However, application in practice still remains unclear despite recent steps taken by the Spanish Ministry of Health $(\mathrm{MoH})$ towards increased transparency in Pricing and Reimbursement (P\&R) decisions: the Interministerial Committee on Pricing of Medicines and Healthcare Products ("CIPM" from Spanish initials), the ultimate P\&R decision-maker, publishes a short summary of decisions to justify P\&R approval or refusal based on current legislation [9].

In May 2013, a major change was introduced in the P\&R process in Spain: The Therapeutic Positioning Report (TPR). The TPR is an evaluation document issued by the Spanish Agency of Medicines and Medical Devices ("AEMPS" from Spanish initials) that aims at determining the adequate positioning relative to what constitutes standard of care for the same indication to inform $P \& R$ decisions in Spain. The TPR includes a thorough review and summary of relative efficacy and safety data available for the new product. The Therapeutic Positioning Coordination Group ("GCPT" from Spanish initials), comprised by the AEMPS, the Directorate-General for the Basic Portfolio of Services of the National Healthcare and Pharmacy System ("DGCBF" from Spanish initials) and the DirectorateGeneral for the Basic Portfolio of Services of the Autonomous Communities (CCAA), coordinates the TPR elaboration as soon as a new drug obtains a positive opinion from the Committee for Medicinal Products for Human Use (CHMP) and begins to work on a draft as soon as the Marketing Authorisation Holder (MAH) ask for P\&R in Spain. This draft is confidential and is shared with the drug's manufacturer and with relevant scientific societies and patient associations so that they can contribute with comments during a one-time allegations phase with a 10 days duration. One the final TPR draft is generated, it is sent to the DGCBF to inform P\&R decision-making. Official TPR elaboration timelines estimate 3 months for the TPR to be drafted. In practice, and based on current metrics, the process can take up to 5 months. The final version of the TPR is published on the AEMPS website only after the $P \& R$ process has been completed and it includes the final $P \& R$ decision. The TPR initiative has contributed in making the $P \& R$ process more transparent but its real impact on the process has not been assessed and remains unclear.

As a result from previous phases of this study, we analysed potential criteria that could drive P\&R decisions for ODs in Spain by analysing ODs approved by the European Commission (EC) between January 2012 and June 2018 that had marketing authorisation in Spain [10]. A total of 64 ODs were included in the analysis, from which only 28 (44.4\%) were reimbursed in Spain and the rest were either undergoing a lengthy decision process or had been rejected. Authors found that a positive TPR conclusion and the existence of no therapeutic alternatives for the evaluated drug were drivers for $P \& R$ approval in Spain, implying that the TPR had become an important step in the Spanish P\&R process.

This study aims to identify ODs authorized in Spain and approved by the EC between January 2003 and December 2019, analyse the impact of the TPR in the P\&R process of ODs in Spain and to assess additional potential criteria that could influence P\&R decisions for ODs.

\section{Results}

Identification of orphan drugs authorised in Spain and approved by the European Commission between 2003 \& 2019 and description of their pricing \& reimbursement situation in Spain

A total of 103 ODs approved by the EC between January 2003 and December 2019 were identified, of which 94 (91.3\%) had been granted marketing authorization in Spain.

Out of the 94 ODs that were authorised in Spain, 46 (48.9\%) had received P\&R approval, 19 (20.2\%) were undergoing the P\&R process and 29 (30.9\%) had their $P \& R$ request rejected. Of these, only 8 ODs were commercialised in the private market: Alprolix ${ }^{\circ}[11]$, Bronchitol $^{\bullet}$ [12], Holoclar ${ }^{\circ}$ [13], Idelvion ${ }^{\bullet}$ [14], NexoBrid ${ }^{\bullet}$ [15], Procysbi $^{\circ}$ [16], Tobi Podhaler ${ }^{\circ}$ [17] and Xermelo ${ }^{\circ}$ [18].

The mean time from EC approval to P\&R approval for ODs in Spain was $20.4 \pm 13.1$ months, with a minimum of 4 months (Kymriah ${ }^{\bullet}$ [19]) and a maximum of 61 months (Revestive [20]). Before the inclusion of the TPR in year 2013, the mean time from EC approval to $P \& R$ approval for ODs in Spain was $25.1 \pm 16.5$. After the inclusion of the TPR during P\&R process in Spain in 
2013, timelines have been reduced by an average of 9 months.

After 2013, the mean regulatory time from EC approval to Spanish marketing authorisation has decreased by an average of 4 months (from $7.5 \pm 10.2$ months in years $2003-2013$ to $3.8 \pm 7.6$ months in years 2014 2019). The inclusion of TPRs during $P \& R$ negotiations in Spain has reduced the mean time from the Spanish marketing authorisation to $P \& R$ approval by an average of 5 months (from 17.3 \pm 13.1 months in years 20032013 to $12.3 \pm 5$ months in years 2014-2019) (Fig. 1).

Clinical and regulatory variables relevant for the pricing \& reimbursement process in Spain

Results from identification of clinical and regulatory variables along with the reimbursement status of each OD are shown in Table 1. From the total of 94 studied ODs authorised in Spain for treating rare diseases, 34 ODs (36.2\%) were indicated for oncologic diseases, 62 ODs (66\%) had already therapeutic alternative indicated for treating the same condition, 57 ODs $(60.6 \%)$ were indicated for rare diseases with a prevalence of $<5 / 10,000$ inhabitants, 38 ODs $(40.4 \%)$ had hard clinical trial outcomes, 53 ODs $(56.4 \%)$ had a superior efficacy profile, 74 ODs $(78.7 \%)$ did not have the obligation by the
European Medicines Agency (EMA) to conduct a Postauthorisation safety study (PASS) and 53 ODs (56.4\%) were indicated for adult patients.

Out of the 53 published TPRs, 42 ODs (79.2\%) had a positive conclusion. From the 94 studied ODs, 84 ODs (89.4\%) were not granted conditional approval marketing authorisation by the EMA (Fig. 2).

Clinical and regulatory variables relevant for the pricing \& reimbursement process in Spain according to its reimbursement status

ODs for which P\&R had been approved

Out of the 46 reimbursed ODs, 24 ODs (52.2\%) were indicated for oncologic diseases, 32 ODs (69.6\%) had a therapeutic alternative, 28 ODs $(60.9 \%)$ were indicated for rare diseases with a prevalence of $<5 / 10,000$ inhabitants, 22 ODs $(47.8 \%)$ had hard clinical trial outcomes, 27 ODs (58.7\%) had a superior efficacy profile, 35 ODs (76.1\%) did not have the obligation by the EMA to conduct a PASS and 31 ODs (67.4\%) were indicated for adult patients.

Out of the 34 ODs with a published TPR, 34 ODs $(100 \%)$ had a positive TPR conclusion. Out of the 46 reimbursed ODs, 43 ODs (93.5\%) were not granted conditional marketing authorisation by the EMA.

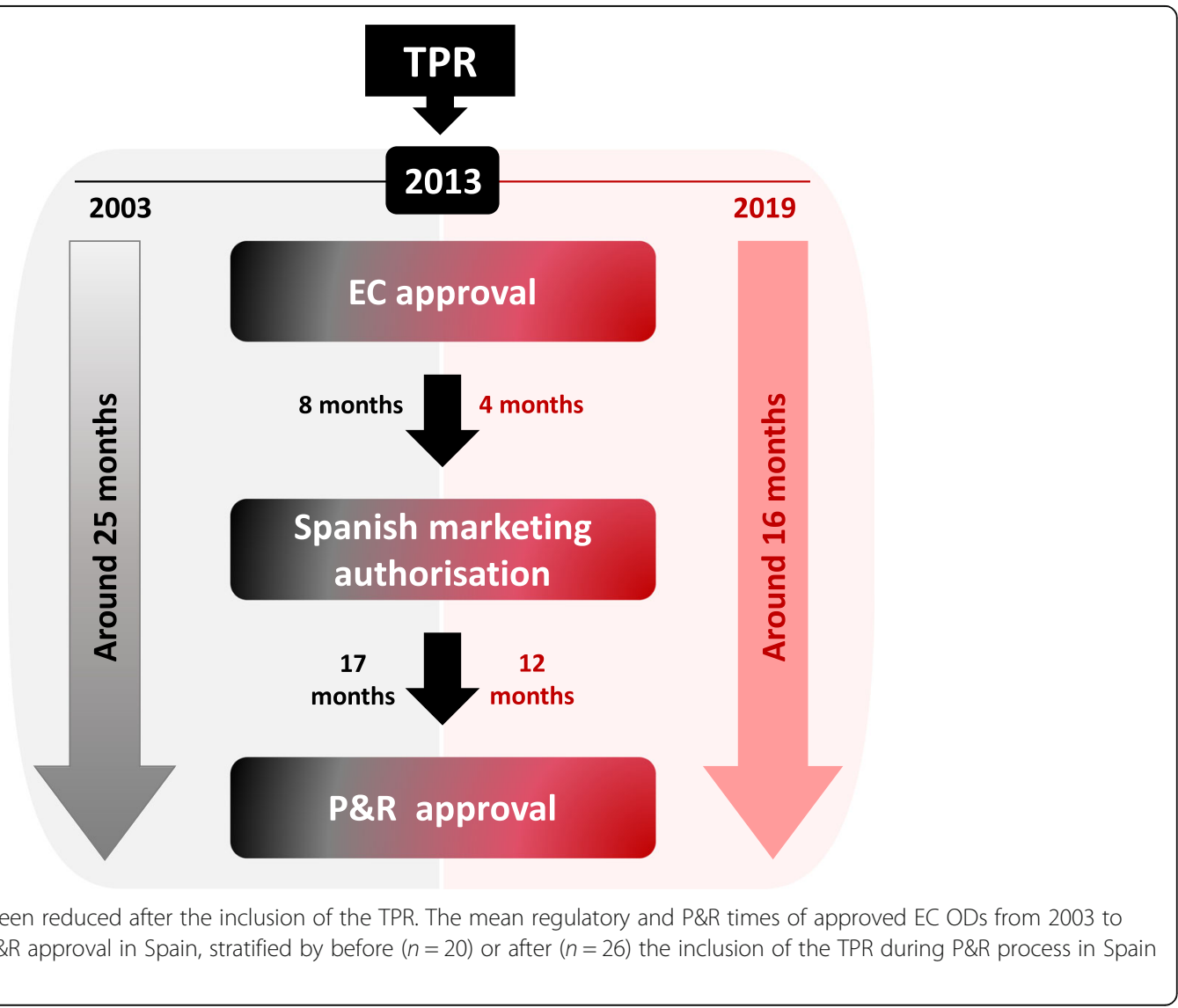


Table 1 Identified variables for each orphan drug authorised in Spain and approved by the European Commission between 2003 \& 2019

\begin{tabular}{|c|c|c|c|c|c|c|c|c|c|c|}
\hline \multirow[t]{2}{*}{ Brand name } & \multirow{2}{*}{$\begin{array}{l}\text { P\&R status } \\
\text { in Spain }\end{array}$} & \multicolumn{7}{|c|}{ Clinical variables } & \multicolumn{2}{|c|}{ Regulatory variables } \\
\hline & & $\begin{array}{l}\text { Therapeutic } \\
\text { Area }\end{array}$ & $\begin{array}{l}\text { Existence of } \\
\text { therapeutic } \\
\text { alternatives }\end{array}$ & $\begin{array}{l}\text { Rarity of } \\
\text { disease }\end{array}$ & $\begin{array}{l}\text { Outcomes } \\
\text { classification }\end{array}$ & $\begin{array}{l}\text { Efficacy } \\
\text { profile }\end{array}$ & $\begin{array}{l}\text { Safety } \\
\text { profile* }\end{array}$ & $\begin{array}{l}\text { Type of } \\
\text { population }\end{array}$ & $\begin{array}{l}\text { TPR } \\
\text { conclusion }\end{array}$ & $\begin{array}{l}\text { Conditional } \\
\text { approval }\end{array}$ \\
\hline Adcetris $^{\oplus}$ & Approved & Oncologic & Yes & $\begin{array}{l}\text { Ultra- } \\
\text { rare }\end{array}$ & Hard & Similar & No & Adults & Positive & Yes \\
\hline Adempas $^{\oplus}$ & Approved & Other & No & $\begin{array}{l}\text { Ultra- } \\
\text { rare }\end{array}$ & Medium & Superior & No & Adults & Positive & No \\
\hline Alofisel $^{\circledast}$ & Approved & Oncologic & Yes & Rare & Soft & Similar & No & Adults & Positive & No \\
\hline Alprolix ${ }^{\oplus}$ & Rejected & Other & Yes & $\begin{array}{l}\text { Ultra- } \\
\text { rare }\end{array}$ & Medium & Similar & No & All ages & Positive & No \\
\hline Besponsa ${ }^{\oplus}$ & Approved & Oncologic & No & Rare & Hard & Superior & No & Adults & Positive & No \\
\hline Blincyto ${ }^{\circledast}$ & Rejected & Oncologic & Yes & Rare & Hard & Similar & Yes & All ages & Positive & No \\
\hline Brineura $^{\oplus}$ & $\begin{array}{l}\text { Under P\&R } \\
\text { decision process }\end{array}$ & Other & No & $\begin{array}{l}\text { Ultra- } \\
\text { rare }\end{array}$ & Medium & Similar & Yes & All ages & $\begin{array}{l}\text { Not } \\
\text { published }\end{array}$ & No \\
\hline Bronchito $^{\oplus}$ & Rejected & Other & Yes & Rare & Medium & Similar & No & All ages & $\begin{array}{l}\text { Not } \\
\text { published }\end{array}$ & No \\
\hline Cablivi $^{\oplus}$ & $\begin{array}{l}\text { Under P\&R } \\
\text { decision process }\end{array}$ & Other & Yes & $\begin{array}{l}\text { Ultra- } \\
\text { rare }\end{array}$ & Soft & Superior & No & Adults & $\begin{array}{l}\text { Not } \\
\text { published }\end{array}$ & No \\
\hline Carbaglu $^{\oplus}$ & Approved & Other & Yes & $\begin{array}{l}\text { Ultra- } \\
\text { rare }\end{array}$ & Soft & Superior & Yes & Adults & $\begin{array}{l}\text { Not } \\
\text { published }\end{array}$ & No \\
\hline Cerdelga ${ }^{\circledast}$ & Approved & Other & Yes & $\begin{array}{l}\text { Ultra- } \\
\text { rare }\end{array}$ & Soft & Superior & Yes & Adults & Positive & No \\
\hline $\begin{array}{l}\text { Chenodeoxycholic } \\
\text { acid Leadiant }{ }^{\circledR}\end{array}$ & $\begin{array}{l}\text { Under P\&R } \\
\text { decision process }\end{array}$ & Other & No & $\begin{array}{l}\text { Ultra- } \\
\text { rare }\end{array}$ & Soft & Similar & No & All ages & $\begin{array}{l}\text { Not } \\
\text { published }\end{array}$ & No \\
\hline Cometriq $^{\oplus}$ & Rejected & Oncologic & Yes & $\begin{array}{l}\text { Ultra- } \\
\text { rare }\end{array}$ & Hard & Similar & No & Adults & Positive & Yes \\
\hline Cresemba $^{\oplus}$ & Approved & Other & Yes & $\begin{array}{l}\text { Ultra- } \\
\text { rare }\end{array}$ & Hard & Similar & No & Adults & Positive & No \\
\hline Crysvita® & $\begin{array}{l}\text { Under P\&R } \\
\text { decision process }\end{array}$ & Other & No & $\begin{array}{l}\text { Ultra- } \\
\text { rare }\end{array}$ & Medium & Similar & Yes & Paediatric & $\begin{array}{l}\text { Not } \\
\text { published }\end{array}$ & Yes \\
\hline Cystadrops $^{\oplus}$ & Rejected & Other & Yes & $\begin{array}{l}\text { Ultra- } \\
\text { rare }\end{array}$ & Soft & Superior & No & All ages & Positive & No \\
\hline Dacogen $^{\circledast}$ & Approved & Oncologic & Yes & Rare & Hard & Superior & No & Adults & Positive & No \\
\hline Darzalex ${ }^{\circledast}$ & Approved & Oncologic & Yes & Rare & Hard & Superior & No & Adults & Positive & No \\
\hline Deltyba® & Approved & Other & Yes & Rare & Soft & Similar & No & Adults & Positive & Yes \\
\hline Epidyolex ${ }^{\oplus}$ & $\begin{array}{l}\text { Under P\&R } \\
\text { decision process }\end{array}$ & Other & Yes & $\begin{array}{l}\text { Ultra- } \\
\text { rare }\end{array}$ & Hard & Similar & No & All ages & $\begin{array}{l}\text { Not } \\
\text { published }\end{array}$ & No \\
\hline Esbriet $^{\oplus}$ & Approved & Oncologic & No & Rare & Medium & Similar & No & Adults & Positive & No \\
\hline Farydak ${ }^{\circledast}$ & Rejected & Oncologic & Yes & Rare & Hard & Similar & No & Adults & Negative & No \\
\hline Firazyr ${ }^{\oplus}$ & Approved & Other & Yes & Rare & Medium & Similar & No & All ages & $\begin{array}{l}\text { Not } \\
\text { published }\end{array}$ & No \\
\hline Firdapse ${ }^{\oplus}$ & Rejected & Other & No & $\begin{array}{l}\text { Ultra- } \\
\text { rare }\end{array}$ & Hard & Superior & No & Adults & $\begin{array}{l}\text { Not } \\
\text { published }\end{array}$ & No \\
\hline Galafold ${ }^{\oplus}$ & Approved & Other & Yes & $\begin{array}{l}\text { Ultra- } \\
\text { rare }\end{array}$ & Soft & Similar & No & Adults & Positive & No \\
\hline Gazyvaro $^{\circledast}$ & Approved & Oncologic & Yes & Rare & Hard & Superior & No & Adults & Positive & No \\
\hline Granupas $^{\oplus}$ & Rejected & Other & No & Rare & Medium & Similar & No & All ages & $\begin{array}{l}\text { Not } \\
\text { published }\end{array}$ & No \\
\hline Holoclar ${ }^{\circledast}$ & Rejected & Other & No & Rare & Hard & Similar & No & All ages & Negative & Yes \\
\hline Iclusig ${ }^{\circledast}$ & Approved & Oncologic & No & Rare & Soft & Similar & Yes & Adults & $\begin{array}{l}\text { Not } \\
\text { published }\end{array}$ & No \\
\hline
\end{tabular}


Table 1 Identified variables for each orphan drug authorised in Spain and approved by the European Commission between 2003 \& 2019 (Continued)

\begin{tabular}{|c|c|c|c|c|c|c|c|c|c|c|}
\hline \multirow[t]{2}{*}{ Brand name } & \multirow{2}{*}{$\begin{array}{l}\text { P\&R status } \\
\text { in Spain }\end{array}$} & \multicolumn{7}{|c|}{ Clinical variables } & \multicolumn{2}{|c|}{ Regulatory variables } \\
\hline & & $\begin{array}{l}\text { Therapeutic } \\
\text { Area }\end{array}$ & $\begin{array}{l}\text { Existence of } \\
\text { therapeutic } \\
\text { alternatives }\end{array}$ & $\begin{array}{l}\text { Rarity of } \\
\text { disease }\end{array}$ & $\begin{array}{l}\text { Outcomes } \\
\text { classification }\end{array}$ & $\begin{array}{l}\text { Efficacy } \\
\text { profile }\end{array}$ & $\begin{array}{l}\text { Safety } \\
\text { profile* }\end{array}$ & $\begin{array}{l}\text { Type of } \\
\text { population }\end{array}$ & $\begin{array}{l}\text { TPR } \\
\text { conclusion }\end{array}$ & $\begin{array}{l}\text { Conditional } \\
\text { approval }\end{array}$ \\
\hline Idelvion ${ }^{\circledast}$ & Rejected & Other & Yes & $\begin{array}{l}\text { Ultra- } \\
\text { rare }\end{array}$ & Hard & Similar & No & All ages & Positive & No \\
\hline Imbruvica ${ }^{\oplus}$ & Approved & Oncologic & Yes & Rare & Hard & Similar & No & Adults & Positive & No \\
\hline Imnovid ${ }^{\oplus}$ & Approved & Oncologic & Yes & Rare & Hard & Superior & Yes & Adults & Positive & No \\
\hline Jorveza ${ }^{\circledR}$ & Rejected & Other & Yes & Rare & Soft & Superior & No & Adults & Negative & No \\
\hline Kalydeco ${ }^{\oplus}$ & Approved & Other & No & Rare & Medium & Similar & No & All ages & Positive & No \\
\hline Kanuma ${ }^{\oplus}$ & Approved & Other & No & $\begin{array}{l}\text { Ultra- } \\
\text { rare }\end{array}$ & Hard & Superior & Yes & All ages & Positive & No \\
\hline Kuvan ${ }^{\oplus}$ & Approved & Other & Yes & Rare & Soft & Superior & No & All ages & $\begin{array}{l}\text { Not } \\
\text { published }\end{array}$ & No \\
\hline Kymriah ${ }^{\oplus}$ & Approved & Oncologic & Yes & Rare & Hard & Similar & Yes & All ages & Positive & No \\
\hline Kyprolis ${ }^{\oplus}$ & Approved & Oncologic & Yes & Rare & Hard & Superior & No & Adults & Positive & No \\
\hline Lamzede $^{\oplus}$ & $\begin{array}{l}\text { Under P\&R } \\
\text { decision process }\end{array}$ & Other & No & $\begin{array}{l}\text { Ultra- } \\
\text { rare }\end{array}$ & Soft & Superior & No & All ages & $\begin{array}{l}\text { Not } \\
\text { published }\end{array}$ & No \\
\hline Ledaga $^{\circledR}$ & Rejected & Oncologic & Yes & Rare & Hard & Superior & No & Adults & Negative & No \\
\hline Lutathera® & Approved & Other & Yes & $\begin{array}{l}\text { Ultra- } \\
\text { rare }\end{array}$ & Hard & Superior & No & Adults & Positive & No \\
\hline Luxturna ${ }^{\circledast}$ & $\begin{array}{l}\text { Under P\&R } \\
\text { decision process }\end{array}$ & Other & No & Rare & Medium & Superior & Yes & All ages & $\begin{array}{l}\text { Not } \\
\text { published }\end{array}$ & No \\
\hline Mepsevii ${ }^{\oplus}$ & $\begin{array}{l}\text { Under P\&R } \\
\text { decision process }\end{array}$ & Other & No & $\begin{array}{l}\text { Ultra- } \\
\text { rare }\end{array}$ & Soft & Similar & No & All ages & $\begin{array}{l}\text { Not } \\
\text { published }\end{array}$ & No \\
\hline Mozobil $^{\circledast}$ & Approved & Oncologic & Yes & Rare & Medium & Superior & No & All ages & $\begin{array}{l}\text { Not } \\
\text { published }\end{array}$ & No \\
\hline Myalepta® & Rejected & Other & Yes & $\begin{array}{l}\text { Ultra- } \\
\text { rare }\end{array}$ & Soft & Similar & No & All ages & $\begin{array}{l}\text { Not } \\
\text { published }\end{array}$ & No \\
\hline Mylotarg & Approved & Oncologic & No & Rare & Hard & Superior & No & All ages & Positive & No \\
\hline Namuscla ${ }^{\oplus}$ & $\begin{array}{l}\text { Under P\&R } \\
\text { decision process }\end{array}$ & Other & No & Rare & Hard & Superior & No & Adults & $\begin{array}{l}\text { Not } \\
\text { published }\end{array}$ & No \\
\hline Natpar ${ }^{\circledast}$ & Rejected & Other & Yes & $\begin{array}{l}\text { Ultra- } \\
\text { rare }\end{array}$ & Soft & Superior & No & Adults & Negative & Yes \\
\hline Nexavar ${ }^{\circledast}$ & Approved & Oncologic & Yes & Rare & Hard & Superior & No & All ages & $\begin{array}{l}\text { Not } \\
\text { published }\end{array}$ & No \\
\hline NexoBrid ${ }^{\circledR}$ & Rejected & Other & No & Rare & Medium & Superior & Yes & Adults & $\begin{array}{l}\text { Not } \\
\text { published }\end{array}$ & No \\
\hline Ninlaro ${ }^{\oplus}$ & Rejected & Oncologic & Yes & Rare & Hard & Superior & No & Adults & Negative & Yes \\
\hline Ocaliva ${ }^{\circledast}$ & Approved & Other & Yes & $\begin{array}{l}\text { Ultra- } \\
\text { rare }\end{array}$ & Soft & Superior & No & Adults & Positive & Yes \\
\hline $\mathrm{Ofev}^{\oplus}$ & Approved & Oncologic & No & Rare & Medium & Similar & No & Adults & Positive & No \\
\hline Onivyde ${ }^{\circledast}$ & Approved & Oncologic & Yes & Rare & Hard & Superior & No & Adults & Positive & No \\
\hline Onpattro ${ }^{\oplus}$ & $\begin{array}{l}\text { Under P\&R } \\
\text { decision process }\end{array}$ & Other & Yes & Rare & Hard & Superior & No & Adults & $\begin{array}{l}\text { Not } \\
\text { published }\end{array}$ & No \\
\hline Opsumit ${ }^{\oplus}$ & Approved & Other & Yes & $\begin{array}{l}\text { Ultra- } \\
\text { rare }\end{array}$ & Hard & Superior & No & Adults & Positive & No \\
\hline Orphacol ${ }^{\circledR}$ & Approved & Other & No & $\begin{array}{l}\text { Ultra- } \\
\text { rare }\end{array}$ & Hard & Similar & No & All ages & $\begin{array}{l}\text { Not } \\
\text { published }\end{array}$ & No \\
\hline Oxervate ${ }^{\oplus}$ & Rejected & Other & No & Rare & Hard & Superior & No & Adults & Negative & No \\
\hline
\end{tabular}


Table 1 Identified variables for each orphan drug authorised in Spain and approved by the European Commission between 2003 \& 2019 (Continued)

\begin{tabular}{|c|c|c|c|c|c|c|c|c|c|c|}
\hline \multirow[t]{2}{*}{ Brand name } & \multirow{2}{*}{$\begin{array}{l}\text { P\&R status } \\
\text { in Spain }\end{array}$} & \multicolumn{7}{|c|}{ Clinical variables } & \multicolumn{2}{|c|}{ Regulatory variables } \\
\hline & & $\begin{array}{l}\text { Therapeutic } \\
\text { Area }\end{array}$ & $\begin{array}{l}\text { Existence of } \\
\text { therapeutic } \\
\text { alternatives }\end{array}$ & $\begin{array}{l}\text { Rarity of } \\
\text { disease }\end{array}$ & $\begin{array}{l}\text { Outcomes } \\
\text { classification }\end{array}$ & $\begin{array}{l}\text { Efficacy } \\
\text { profile }\end{array}$ & $\begin{array}{l}\text { Safety } \\
\text { profile* }\end{array}$ & $\begin{array}{l}\text { Type of } \\
\text { population }\end{array}$ & $\begin{array}{l}\text { TPR } \\
\text { conclusion }\end{array}$ & $\begin{array}{l}\text { Conditional } \\
\text { approval }\end{array}$ \\
\hline Palynziq $^{\circledast}$ & $\begin{array}{l}\text { Under P\&R } \\
\text { decision process }\end{array}$ & Other & Yes & Rare & Soft & Superior & Yes & All ages & $\begin{array}{l}\text { Not } \\
\text { published }\end{array}$ & No \\
\hline Plenadren $^{\oplus}$ & Rejected & Other & Yes & Rare & Soft & Similar & No & Adults & $\begin{array}{l}\text { Not } \\
\text { published }\end{array}$ & No \\
\hline Poteligeo $^{\oplus}$ & $\begin{array}{l}\text { Under P\&R } \\
\text { decision process }\end{array}$ & Oncologic & Yes & Rare & Hard & Superior & No & Adults & $\begin{array}{l}\text { Not } \\
\text { published }\end{array}$ & No \\
\hline Prevymis $^{\oplus}$ & Rejected & Other & Yes & Rare & Medium & Superior & No & Adults & Negative & No \\
\hline Procysbi ${ }^{\circledast}$ & Rejected & Other & Yes & $\begin{array}{l}\text { Ultra- } \\
\text { rare }\end{array}$ & Soft & Similar & No & All ages & Negative & No \\
\hline Qarziba ${ }^{\oplus}$ & $\begin{array}{l}\text { Under P\&R } \\
\text { decision process }\end{array}$ & Oncologic & Yes & Rare & Soft & Similar & Yes & Paediatric & $\begin{array}{l}\text { Not } \\
\text { published }\end{array}$ & No \\
\hline Ravicti ${ }^{\circledast}$ & Approved & Other & Yes & Rare & Soft & Similar & Yes & All ages & $\begin{array}{l}\text { Not } \\
\text { published }\end{array}$ & No \\
\hline Raxone $e^{\oplus}$ & $\begin{array}{l}\text { Under P\&R } \\
\text { decision process }\end{array}$ & Other & No & Rare & Medium & Similar & No & All ages & $\begin{array}{l}\text { Not } \\
\text { published }\end{array}$ & No \\
\hline Revestive $^{\circledast}$ & Approved & Other & No & $\begin{array}{l}\text { Ultra- } \\
\text { rare }\end{array}$ & Medium & Superior & Yes & Adults & $\begin{array}{l}\text { Not } \\
\text { published }\end{array}$ & No \\
\hline Rydapt $^{\oplus}$ & Approved & Oncologic & Yes & $\begin{array}{l}\text { Ultra- } \\
\text { rare }\end{array}$ & Hard & Superior & No & Adults & Positive & No \\
\hline Scenesse ${ }^{\oplus}$ & Rejected & Other & No & $\begin{array}{l}\text { Ultra- } \\
\text { rare }\end{array}$ & Soft & Superior & No & Adults & $\begin{array}{l}\text { Not } \\
\text { published }\end{array}$ & No \\
\hline Signifor ${ }^{\circledR}$ & Approved & Other & Yes & Rare & Soft & Superior & No & Adults & Positive & No \\
\hline Sirturo $^{\oplus}$ & Rejected & Other & Yes & Rare & Soft & Superior & No & Adults & Positive & Yes \\
\hline Soliris ${ }^{\circledast}$ & Approved & Oncologic & Yes & Rare & Medium & Superior & No & All ages & Positive & No \\
\hline SomaKit TOC ${ }^{\circledast}$ & Approved & Other & Yes & Rare & Soft & Similar & No & Adults & Positive & No \\
\hline Spinraza ${ }^{\oplus}$ & Approved & Other & No & $\begin{array}{l}\text { Ultra- } \\
\text { rare }\end{array}$ & Hard & Superior & No & All ages & Positive & No \\
\hline Strensiq ${ }^{\circledast}$ & Rejected & Other & No & $\begin{array}{l}\text { Ultra- } \\
\text { rare }\end{array}$ & Soft & Similar & No & Paediatric & Positive & No \\
\hline Sylvant ${ }^{\circledast}$ & Approved & Oncologic & No & Rare & Medium & Superior & Yes & Adults & Positive & No \\
\hline Symkevi ${ }^{\oplus}$ & Approved & Other & Yes & Rare & Medium & Superior & No & All ages & Positive & No \\
\hline Takhzyro ${ }^{\oplus}$ & $\begin{array}{l}\text { Under P\&R } \\
\text { decision process }\end{array}$ & Other & Yes & $\begin{array}{l}\text { Ultra- } \\
\text { rare }\end{array}$ & Medium & Superior & No & All ages & $\begin{array}{l}\text { Not } \\
\text { published }\end{array}$ & No \\
\hline Tegsedi ${ }^{\oplus}$ & $\begin{array}{l}\text { Under P\&R } \\
\text { decision process }\end{array}$ & Other & Yes & Rare & Medium & Superior & No & Adults & $\begin{array}{l}\text { Not } \\
\text { published }\end{array}$ & No \\
\hline Tepadina ${ }^{\oplus}$ & Approved & Oncologic & Yes & $\begin{array}{l}\text { Ultra- } \\
\text { rare }\end{array}$ & Hard & Similar & No & All ages & $\begin{array}{l}\text { Not } \\
\text { published }\end{array}$ & No \\
\hline Tobi Podhaler ${ }^{\oplus}$ & Rejected & Other & Yes & Rare & Medium & Superior & No & All ages & $\begin{array}{l}\text { Not } \\
\text { published }\end{array}$ & No \\
\hline Translarna ${ }^{\circledast}$ & Rejected & Other & No & Rare & Medium & Similar & No & All ages & Negative & Yes \\
\hline Verkazia ${ }^{\oplus}$ & $\begin{array}{l}\text { Under P\&R } \\
\text { decision process }\end{array}$ & Other & Yes & Rare & Soft & Superior & No & Paediatric & $\begin{array}{l}\text { Not } \\
\text { published }\end{array}$ & No \\
\hline Vimizim ${ }^{\oplus}$ & Rejected & Other & No & $\begin{array}{l}\text { Ultra- } \\
\text { rare }\end{array}$ & Medium & Superior & Yes & All ages & Negative & No \\
\hline Votubia ${ }^{\circledR}$ & Approved & Oncologic & No & Rare & Medium & Superior & No & Adults & $\begin{array}{l}\text { Not } \\
\text { published }\end{array}$ & No \\
\hline Vpriv $^{\oplus}$ & Approved & Other & Yes & $\begin{array}{l}\text { Ultra- } \\
\text { rare }\end{array}$ & Soft & Similar & No & All ages & $\begin{array}{l}\text { Not } \\
\text { published }\end{array}$ & No \\
\hline
\end{tabular}


Table 1 Identified variables for each orphan drug authorised in Spain and approved by the European Commission between 2003 \& 2019 (Continued)

\begin{tabular}{|c|c|c|c|c|c|c|c|c|c|c|}
\hline \multirow[t]{2}{*}{ Brand name } & \multirow{2}{*}{$\begin{array}{l}\text { P\&R status } \\
\text { in Spain }\end{array}$} & \multicolumn{7}{|c|}{ Clinical variables } & \multicolumn{2}{|c|}{ Regulatory variables } \\
\hline & & $\begin{array}{l}\text { Therapeutic } \\
\text { Area }\end{array}$ & $\begin{array}{l}\text { Existence of } \\
\text { therapeutic } \\
\text { alternatives }\end{array}$ & $\begin{array}{l}\text { Rarity of } \\
\text { disease }\end{array}$ & $\begin{array}{l}\text { Outcomes } \\
\text { classification }\end{array}$ & $\begin{array}{l}\text { Efficacy } \\
\text { profile }\end{array}$ & $\begin{array}{l}\text { Safety } \\
\text { profile* }\end{array}$ & $\begin{array}{l}\text { Type of } \\
\text { population }\end{array}$ & $\begin{array}{l}\text { TPR } \\
\text { conclusion }\end{array}$ & $\begin{array}{l}\text { Conditional } \\
\text { approval }\end{array}$ \\
\hline Vyndaqe $^{\circledast}$ & Approved & Other & No & $\begin{array}{l}\text { Ultra- } \\
\text { rare }\end{array}$ & Medium & Similar & Yes & Adults & Positive & No \\
\hline$V_{y x e o s}$ & Rejected & Oncologic & Yes & Rare & Hard & Superior & No & Adults & $\begin{array}{l}\text { Not } \\
\text { published }\end{array}$ & No \\
\hline Wakix ${ }^{\oplus}$ & Rejected & Other & Yes & Rare & Hard & Similar & Yes & Adults & Positive & No \\
\hline Xaluprine ${ }^{\oplus}$ & $\begin{array}{l}\text { Under P\&R } \\
\text { decision process }\end{array}$ & Oncologic & Yes & Rare & Soft & Similar & No & All ages & $\begin{array}{l}\text { Not } \\
\text { published }\end{array}$ & No \\
\hline Xermelo ${ }^{\oplus}$ & Rejected & Other & Yes & Rare & Soft & Superior & No & Adults & $\begin{array}{l}\text { Not } \\
\text { published }\end{array}$ & No \\
\hline Xospata ${ }^{\circledast}$ & $\begin{array}{l}\text { Under P\&R } \\
\text { decision process }\end{array}$ & Oncologic & No & Rare & Hard & Superior & No & Adults & $\begin{array}{l}\text { Not } \\
\text { published }\end{array}$ & No \\
\hline Yescarta ${ }^{\oplus}$ & Approved & Oncologic & Yes & Rare & Hard & Similar & Yes & Adults & Positive & No \\
\hline Zejula ${ }^{\oplus}$ & Approved & Oncologic & Yes & $\begin{array}{l}\text { Ultra- } \\
\text { rare }\end{array}$ & Hard & Superior & No & Adults & Positive & No \\
\hline
\end{tabular}

P\&R Pricing and reimbursement; TPR Therapeutic positioning report. *Obligation or not to conduct a post-authorisation safety study (PASS)

\section{ODs for which P\&R had been rejected}

Out of the 29 P\&R rejected ODs, 6 ODs (20.7\%) were indicated for oncologic diseases, 20 ODs (69\%) had a therapeutic alternative, 18 ODs (62.1\%) were indicated for rare diseases with a prevalence of $<5 / 10,000$ inhabitants, 11 ODs (37.9\%) had hard clinical trial outcomes, 15 ODs (51.7\%) had a superior efficacy profile, 25 ODs (86.2\%) did not have the obligation by the EMA to conduct a PASS and 16 ODs (55.2\%) were indicated for adult patients.

Out of the 19 ODs (65.5\%) with a published TPR, 8 ODs (42\%) had a positive TPR conclusion. Out of the 29 ODs without reimbursement, 23 ODs (79.3\%) were not granted conditional marketing authorisation by the EMA.

\section{Analysis of potential relationship between clinical and regulatory variables and reimbursement status of ODs in Spain}

The objective of the analysis was to test the validity of hypotheses described in the methodology section, and to identify variables that may positively influence reimbursement in Spain. A logistic regression model was used to predict the impact of the studied variables on reimbursement: existence of a therapeutic alternative, outcomes classification, efficacy profile, safety profile, TPR conclusion and conditional approval. The analysis included P\&R approved and P\&R rejected ODs in Spain $(n=75)$.

The TPR conclusion variable was clearly correlated to the reimbursement status for ODs in Spain $(p$-value $<0$, 0001). As a result, ODs with a positive TPR conclusion (ODs recommended for a group of patients or considered equivalent to an alternative approved in Spain) would be more likely to be reimbursed in Spain. The regression analysis results did not include the TPR conclusion variable due to perfect separation. The results of the univariate and multivariate regression model did not show a significant correlation between the studied variables and reimbursement status (Table 2).

The variable that has the largest impact on the probability of reimbursement in Spain is the TPR conclusion. This means that ODs with a positive TPR conclusion are more likely to be reimbursed.

\section{Discussion}

A total of 94 ODs have been approved by the EC between January 2003 and December 2019 and have marketing authorisation in Spain. The mean time from EC approval to P\&R approval for ODs in Spain was 20.4 \pm 13.1 months and the mean time from Spanish marketing authorisation to $\mathrm{P} \& \mathrm{R}$ approval was $14 \pm 9.74$ months. Based on the results of the study, having the EC and Spanish marketing authorisation approval does not guarantee access within the Spanish market, as from the 94 studied ODs, 46 (48.9\%) were reimbursed in Spain at the moment of the study, and the rest of ODs were either undergoing the decision process or rejected, which prevents patients equitable and timely access to these drugs.

This study shows that the only studied variable that seems to affect $P \& R$ decisions in Spain is the conclusion of the TPR. This variable has been the only are consistently showing statistical significance across different analyses of the present study considering different time 


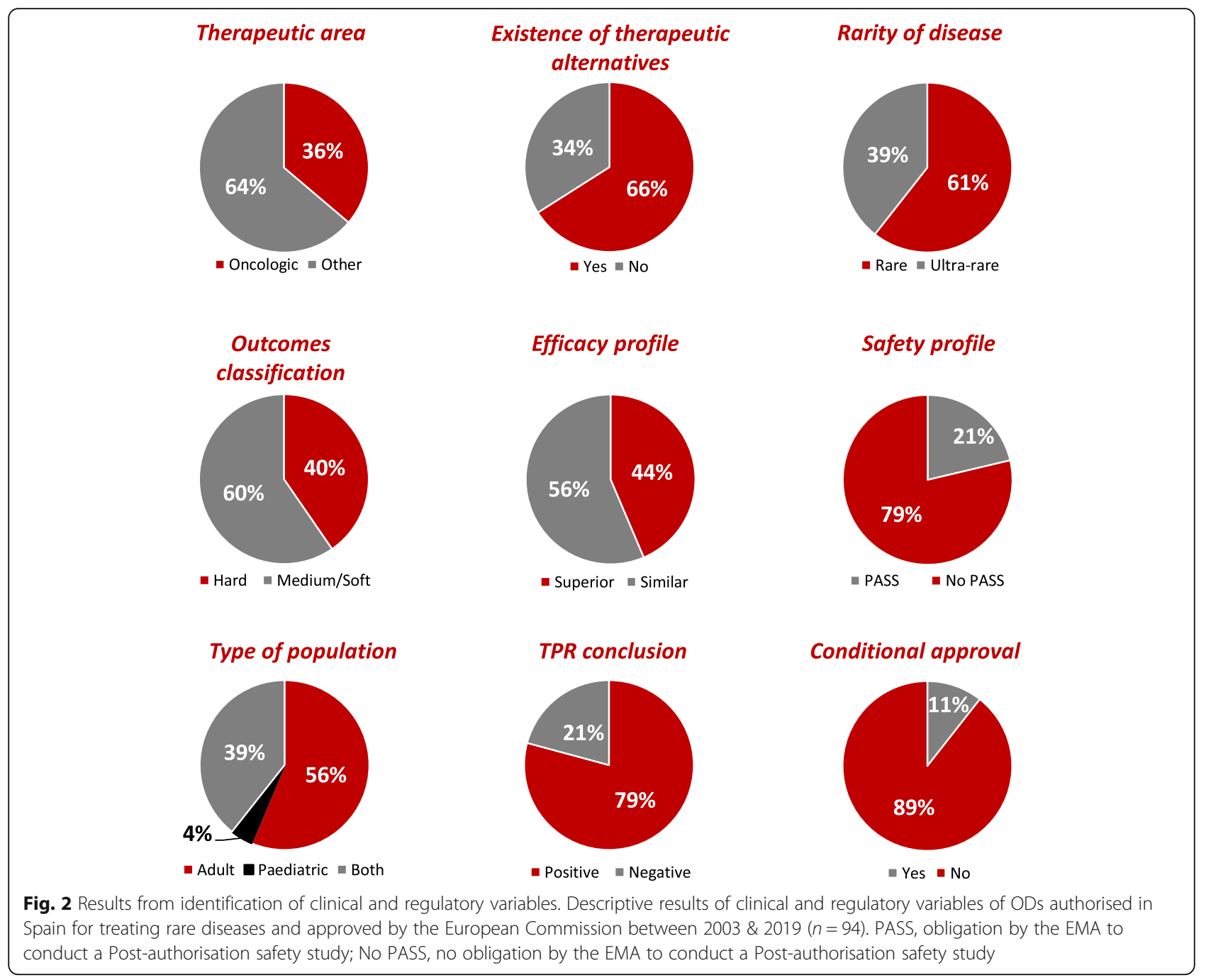

Table 2 Results of the regression analysis

\begin{tabular}{llllll}
\hline Variable & Estimate & $95 \% \mathrm{Cl}$ & $|\mathrm{Z}|$ & $\boldsymbol{P}$ value & $\boldsymbol{P}$ value classification \\
\hline Univariate regression analysis & & & & & \\
Existence of therapeutic alternative & 1,029 & 0,3674 to 2,798 & 0,05485 & 0,9563 & $\mathrm{~ns}$ \\
Outcomes classification & 1,5 & 0,5863 to 3,943 & 0,8389 & 0,4015 & $\mathrm{~ns}$ \\
$\begin{array}{l}\text { Efficacy profile } \\
\text { Safety profile }\end{array}$ & 0,754 & 0,2938 to 1,927 & 0,5917 & 0,554 & $\mathrm{~ns}$ \\
Conditional approval & 1,964 & 0,5944 to 7,735 & 1,055 & 0,2914 & $\mathrm{~ns}$ \\
Multivariate regression analysis & 0,2674 & 0,0525 to 1,111 & 1,752 & 0,0798 & $\mathrm{~ns}$ \\
$\begin{array}{l}\text { Existence of therapeutic alternative } \\
\text { Outcomes classification }\end{array}$ & 1,129 & 0,3821 to 3,280 & 0,2231 & 0,8234 & $\mathrm{~ns}$ \\
Efficacy profile & 1,518 & 0,57 to 4,165 & 0,8289 & 0,4072 & $\mathrm{~ns}$ \\
Safety profile & 0,8212 & 0,3092 to 2,193 & 0,3968 & 0,6915 & $\mathrm{~ns}$ \\
Conditional approval & 1,731 & 0,4964 to 7,141 & 0,8238 & 0,41 & $\mathrm{~ns}$ \\
\hline
\end{tabular}

The dependent variable in logistic regression was reimbursement status, stratified by P\&R approved or P\&R rejected $(n=75)$. Cl Confidence interval; $Z Z$-score; ns: not significant 
periods [10], demonstrating the key role that the TPR plays in the $P \& R$ process. It is important to highlight that the TPR, since its introduction in 2013, has also contributed to reduce $P \& R$ approval timelines in Spain by an average of 9 months. After 2013, the mean regulatory time from EC approval to Spanish marketing authorisation has decreased nearly 4 months, indicating that the MAH could be asking for $P \& R$ in Spain sooner than in the past and the administrative process might have been accelerated. Authors do not think that the reduction in regulatory timelines from EC approval to Spanish marketing authorisation is related to the introduction of the TPR. Additionally, the introduction of the TPR during P\&R negotiations in Spain could be associated with a reduction of the mean time from the Spanish marketing authorisation to $P \& R$ approval by an average of 5 months.

A recent study that assessed the access to orphan medicines in Spain until September 2019 [21] has reported similar findings to the present study in terms of the identified OD sample, their P\&R status and estimated regulatory times during the $P \& R$ process, thus reinforcing the validity of the data presented in this study. However, the abovementioned study did not assess the potential influence of different variables in $P \& R$ decisions.

None of clinical variables related to $P \& R$ criteria set in the Spanish legislation have been found to directly affect $P \& R$ decisions. This reinforces the widely held opinion in the published literature highlighting the lack of transparency and availability of information with regards to which criteria are used in real life for $P \& R$ evaluation and decision-making of ODs across European countries $[4,6]$.

In recent years, actions have been made at international level to try to reduce uncertainty surrounding the appraisal of ODs and to increase the process' transparency, like the creation of specific frameworks to assess ODs [22] or the publication of recommendations on principles to help improve the consistency of ODs P\&R assessment in Europe [23]. A recent publication from Paulden et al. [22] identified decision criteria that could influence $P \& R$ of ODs, such as the availability of therapeutic alternatives, the evidence of clinical efficacy, the severity of the disease or the impact of treatment on life expectancy and quality of life. Another highlighted point by Paulden et al. [22] is the diversity of views around P\&R decision criteria, depending on the context. Therefore, it would be important and beneficial to incorporate preferences from several stakeholders when making $P \& R$ decisions.

The recent creation of specific frameworks for OD appraisal, including the recent creation of a framework for the evaluation of ODs in Spain at national level $[8,24]$ using Multiple-Criteria Decision Analysis (MCDA) methodology [25], could contribute to ensuring a systematic and transparent evaluation process for ODs'
$P \& R$, aligned with the criteria set in the Spanish legislation, and incorporate preferences from several stakeholders when making P\&R decisions.

Although there is still a long way to go towards total transparency in the P\&R process in Europe and Spain, it should be noted that important advances have been made in recent years that have shed some light and contributing to optimise the $P \& R$ process for innovative medicines in Spain. Important examples include the introduction of the TPR, which incorporates different stakeholder perspectives and has reduced the average time of the $P \& R$ process and has made the Spanish $\mathrm{MoH}$ clinical positioning on the evaluated drug transparent and publicly available. Another important achievement is the publication of the CIPM agreements, where the favourable and unfavourable P\&R decisions are related to the criteria contemplated in the Spanish legislation justifying the favourable or non-favourable decision. Also, the BIFIMED database launched in 2019 [26], which publishes all information regarding the reimbursement of a medicine (except its price), thanks to which we have been able to improve the quality of the data related to $P \& R$ approval times with respect to our previous study [10], where we had to collect this information using indirect sources.

\section{Study limitations}

The studied variables might not take into account all the P\&R criteria that Spanish evaluators take into consideration, such as severity of the disease, unmet needs of specific populations, therapeutic and social drug value, incremental clinical benefit taking into account costeffectiveness, budget impact, existence of alternative treatment options for the indication and degree of innovation, because of the difficulty in comparing and classifying using uniform criteria all the different types of ODs and rare diseases.

Important economic variables that could affect $P \& R$ decisions, like drug price and budget impact (BI) could not be assessed due to the lack of validity of the information available regarding real-life reimbursement. First, the $P \& R$ process is not fully transparent in Spain and the sales forecasts that the manufacturers send to the Ministry of Health are not publicly available. Second, the exact number of patients eligible for each OD's indication was not known. Prevalence data for the majority of rare diseases is not known and, in many cases, there is no published prevalence for the OD's exact indication.

There was not enough power in the study for further analysis. A future analysis of clinical and regulatory variables involving a larger sample size of ODs authorised in Spain is needed to further explore their impact on the $P \& R$ process. 


\section{Conclusions}

From all ODs approved by the EC and which had obtained Marketing Authorisation in Spain, 46 (48.9\%) were reimbursed, 19 (20.2\%) were undergoing decision and $29(30.9 \%)$ were rejected. The Spanish regulatory timelines for ODs have been reduced after the inclusion of the TPR during P\&R process by an average of 9 months. The mean regulatory time from EC approval to Spanish marketing authorisation has decreased by an average of 4 months and the mean time from the Spanish marketing authorisation to $\mathrm{P} \& \mathrm{R}$ approval has decreased by an average of 5 months. A positive TPR conclusion is a key driver for $P \& R$ approval for ODs. In addition, oncology ODs might be more likely to be reimbursed in Spain. Economic variables such as the price of the drug and the total budget impact derived from its introduction were not assessed in this study because of lack of transparency and lack of validity of publicly available information. Official listed prices in the available databases do not reflect the reimbursement price agreed between the Ministry of health and the MAH.

\section{Methods}

A protocol, including an analysis plan, was developed before the development of the present study, which includes the study's hypothesis, variables (and stratification of variables) and pre-specified statistical analyses.

All collected data and statistical analyses were included in an internal data base.

\section{Identification of European Commission approved orphan drugs between 2003 \& 2019}

ODs to treat rare diseases with current orphan designations were retrieved from the European Community Register of orphan medicinal products [27]. ODs with orphan designations that have been expired or removed by the sponsor were excluded from the study. EC approved ODs and their EC approval dates were extracted from the EMA's website [28] through their online medicine finder engine, with the following search filters: human medicines, orphan medicines and authorized medicines. The ODs found were grouped according to the EC authorization year 2003 to 2019.

\section{Identification of orphan drugs authorised in Spain and their pricing \& reimbursement situation}

ODs authorised in Spain were retrieved from the Spanish Medicine Online Information Centre (CIMA) of the AEMPS [29]. This study only included drugs that had been granted marketing authorization in Spain. The CIMA database was used to search if the OD had a Spanish marketing authorisation, its authorisation date and commercialisation status in Spain. The Spanish marketing authorisation dates were used to analyse the time from EC approval to Spanish marketing authorisation and the time from Spanish marketing authorisation to $P \& R$ approval date.

The BIFIMED database was used to search each OD $P \& R$ status information [26]. ODs were classified into three reimbursement categories: $P \& R$ approved (ODs that have had their $P \& R$ request approved), under $P \& R$ decision process (ODs that have requested $P \& R$ but are still under $P \& R$ negotiations) and $P \& R$ rejected (ODs that have seen their $P \& R$ request rejected). $P \& R$ approval dates were used to analyse the time from the Spanish marketing authorisation to $P \& R$ approval in Spain.

\section{Identification and description of relevant variables for the pricing \& reimbursement process in Spain Clinical variables}

Clinical variables were identified based on formal and informal criteria used in the Spanish P\&R process [30-33], which were tested in previous phases of this study [10]. The studied clinical variables are part of the mandatory clinical information that the MAH must provide to the Spanish P\&R regulation bodies: (I) Therapeutic area (ODs were divided into two groups, oncology or other, according to their indication and taking in consideration the Anatomical Therapeutic Chemical (ATC) code), (II) Existence of therapeutic alternatives (ODs were classified into two groups depending on whether a therapeutic alternative was available, known as drugs indicated for treating the same condition), (III) Rarity of disease (Indication's prevalence was analysed and categorised into rare diseases, that affect $<5 / 10,000$ inhabitants, or ultrarare diseases, that affect $<1 / 50,000$ inhabitants. Spanish prevalence data were used when available), (IV) Outcomes classification (The classification of clinicianreported outcomes assessment (COAs) proposed by Powers III, JH. et al. was used [34]. ODs clinical trial outcomes were analysed and classified into hard (measures of survival and patient reported outcomes, PRO), intermedium (functional capacity tests and other clinical reported outcomes, $\mathrm{CRO}$ ) or soft (outcomes assessments using biomarkers)), (V) Efficacy profile (ODs clinical trials were analysed and classified into similar (trial uncontrolled or statistically significantly non-superior efficacy compared with placebo and non-superior efficacy compared with active comparator) or superior (statistically significantly superior efficacy compared with placebo and statistically significantly superior efficacy compared with active comparator) efficacy profile), (VI) Safety profile (ODs were classified into two groups depending on whether they had the obligation by the EMA to conduct a Post-authorisation safety study, PASS) and (VII) Type of population (Categorised into paediatric, adults or both). 
Hypothesis were defined for the following clinical variables: (I) Existence of therapeutic alternatives (ODs indicated for a disease without any therapeutic alternative would be more likely to have $P \& R$ approval, as these patients present high unmet clinical needs compared to patients that can be currently treated), (II) Outcomes classification (ODs with hard outcomes would be more likely to have $P \& R$ approval than drugs with intermedium or soft outcomes since they will represent less uncertainty for evaluators and decision makers), (III) Efficacy profile (ODs that showed a superior efficacy profile would be more likely to be reimbursed) and (IV) Safety profile (ODs that showed a safety profile that raised less uncertainty among evaluators would be more likely to be reimbursed).

The clinical variables (therapeutic area, existence of therapeutic alternatives and rarity of disease) were extracted from the corresponding TPR [35] and/ or the European public assessment report (EPAR) [28]. When no information was available on the prevalence of the diseases, a search in biomedical databases and/ or grey literature was performed and prevalence data was extracted from published epidemiology studies. Outcomes classification, efficacy profile, safety profile and type of population were extracted from the clinical trials mentioned in the EPAR [28].

\section{Regulatory variables}

Regulatory variables were identified, based on formal and informal criteria used in the Spanish $P \& R$ process [30-33] which were tested in previous phases of this study [10]. The studied regulatory variables are part of the regulatory process in Europe or Spain: (I) TPR conclusion (ODs TPR conclusions were analysed and then categorised into positive (ODs recommended to a group of patients or equivalent to an alternative approved in Spain), negative conclusion (ODs not recommended) or missing data (ODs authorized before May 2013 or with their TPR in process)) and (II) Conditional approval (ODs may be granted a conditional marketing authorisation by the EMA. ODs were classified into two groups depending on whether they had a conditional marketing authorisation or not).

Hypothesis were defined for the following regulatory variables: (I) TPR conclusion (ODs with a published TPR with a positive conclusion would be more likely to be reimbursed in Spain) and (VI) Conditional approval (ODs with a conditional approval by the EMA would be less likely to be reimbursed in Spain).

The AEMPS's webpage was used to search information about the publication of the TPR for each OD [35]. The AEMPS started elaborating TPRs in 2013, hence, not all ODs in this study had a TPR available driving the P\&R decision process. The EMA's website [28] was used to search the conditional approval status for each OD.

\section{Statistical analysis}

A descriptive analysis of the clinical and regulatory variables was conducted and further stratified by P\&R status including ODs authorised in Spain and approved by the EC between 2003 and 2019. Quantitative data (including time from EC approval to Spanish marketing authorisation, from Spanish marketing authorisation to P\&R approval and time from Spanish marketing authorisation to $P \& R$ decision process to $31 / 12 / 2019$ ) were described through basic statistic descriptive analysis. Qualitative data were described and stratified by $P \& R$ status.

In order to answer our hypothesis, a statistical analysis of the clinical and regulatory variables was conducted. The objective of the analysis was to test the validity of the previous hypothesis and to identify variables that may positively influence the reimbursement in Spain. A logistic regression model [36] was used to predict the impact of the studied variables on reimbursement in Spain: existence of therapeutic alternatives, outcomes classification, efficacy profile, safety profile, TPR conclusion and conditional approval. Each variable was analysed using an univariate regression model. Additionally, variables were analysed using a multivariate regression model. The analysis included $P \& R$ reimbursed and $P \& R$ rejected ODs in Spain $(n=75)$.

The software used to conduct all statistical analysis was GraphPad Prism 8 (Version 8.3.0).

\section{Abbreviations}

AEMPS: Spanish Agency of Medicines and Medical Devices; ATC: Anatomical Therapeutic Chemical; BI: Budget Impact; CCAA: Autonomous communities; CHMP: Committee for Medicinal Products for Human Use; CIMA: Spanish Medicine Online Information Centre; CIPM: Interministerial Committee on Pricing of Medicines and Healthcare Products; CRO: Clinical Reported Outcome; DGCBF: Directorate-General for the Basic Portfolio of Services of the National Health and Pharmacy System; EC: European Commission; EMA: European Medicines Agency; EPAR: European Public Assessment Report; GCPT: Therapeutic Positioning Coordination Group; HTA: Health Technology Assessment; MAH: Marketing Authorisation Holder; MCDA: Multiple-Criteria Decision Analysis; MoH: Ministry of Health; OD: Orphan Drug; P\&R: Pricing and Reimbursement; PASS: Post-authorisation Safety Study; PRO: Patient Reported Outcome; TPR: Therapeutic Positioning Report

\section{Acknowledgements}

Not applicable.

\section{Authors' information (optional)}

Not applicable.

\section{Authors' contributions}

$X$ Badia was the major contributor in designing the study, designing the protocol, interpreted the data, validated the analysis plan and revised the manuscript. T Vico and J Shepherd created the internal database used in the study, contributed to develop the analysis plan, analysed and interpreted the data and were major contributors in writing the manuscript. A Gil, JL

Poveda-Andrés and C Hernández interpreted the data and were major contributors in revising the manuscript. All authors read and approved the final manuscript. 


\section{Funding}

No funding.

\section{Availability of data and materials}

In 2017, Omakase Consulting S.L. developed an OD database to collect data related to medicinal products with OD designation, currently authorised in Europe and their P\&R situation in Spain. The datasets used and analysed during the current study are available from the corresponding author on reasonable request.

\section{Ethics approval and consent to participate}

Not applicable. The study did not involve human participants (patients or otherwise). In 2017, Omakase Consulting S.L. developed an OD database to collect data related to medicinal products with OD designation, currently authorised in Europe and their P\&R situation in Spain. The study was conducted by analysing data from the mentioned database. The study does not require ethics approval or otherwise approval since it does not involve the participation of patients or the enquiry/analysis of medical records.

\section{Consent for publication}

Not applicable. The study did not contain data from any individual person.

\section{Competing interests}

The authors declare that they have no competing interests.

\section{Author details}

'Omakase Consulting S.L., Barcelona, Spain. ${ }^{2}$ Hospital Universitario y Politécnico de La Fe, Valencia, Spain. ${ }^{3}$ Agencia Española de Medicamentos y Productos Sanitarios, Madrid, Spain.

\section{Received: 31 March 2020 Accepted: 9 August 2020}

\section{Published online: 28 August 2020}

\section{References}

1. Kesselheim AS, Myers JA, Avorn J. Characteristics of clinical trials to support approval of orphan vs nonorphan drugs for cancer. JAMA. 2011;305:2320-6 [cited 2020 Feb 20] Available from: http://www.ncbi.nlm.nih.gov/ pubmed/21642684.

2. Morel T, Arickx F, Befrits G, Siviero P, Van Der Meijden C, Xoxi E, et al. Reconciling uncertainty of costs and outcomes with the need for access to orphan medicinal products: a comparative study of managed entry agreements across seven European countries. Orphanet J Rare Dis. 2013;8.

3. Van den Aardweg S. HTA of orphan drugs across six countries: fair, flawed or failing? Euro Obs. 2010;12:14-7.

4. Simoens S. Pricing and reimbursement of orphan drugs: The need for more transparency [Internet]. Orphanet J. Rare Dis. 2011 [cited 2020 Feb 20]. p. 177. Available from: http://www.2020health.org/2020health/Publications/ publications-2012/Orphan-Drugs.html\%5Cnhttp://www.ingesa.msssi.gob.es/ estadEstudios/documPublica/pdf/atDomiciliaria.pdf\%5Cnhttp://www.ojrd. com/content $/ 6 / 1 / 42$

5. Detiček A, Locatelli I, Kos M. Patient Access to Medicines for Rare Diseases in European Countries. Value Heal [Internet]. Elsevier Ltd; 2018;21:553-560. [cited 2020 Feb 20] Available from: http://www.ncbi.nlm.nih.gov/pubmed/29753352.

6. Zamora B, Maignen F, O'Neill P, Mestre-Ferrandiz J, Garau M. Comparing access to orphan medicinal products in Europe. Orphanet $J$ Rare Dis [Internet]. BioMed Central Ltd. 2019;14:95 [cited 2020 Feb 20] Available from: https://ojrd.biomedcentral.com/articles/10.1186/s13023-019-1078-5.

7. Información sobre Enfermedades Raras | FEDER [Internet]. [cited 2020 Feb 20]. Available from: https://www.enfermedades-raras.org/index.php/ enfermedades-raras.

8. Badia X, Chugani D, Abad MR, Arias P, Guillén-Navarro E, Jarque I, et al. Development and validation of an MCDA framework for evaluation and decision-making of orphan drugs in Spain. Expert Opin. Orphan Drugs. Taylor and Francis Ltd. 2019:363-72.

9. Ministerio de Sanidad, Consumo y Bienestar Social. [Internet]. Com. INTERM INISTERIAL PRECIOS Medicam. Y Prod. Sanit. [cited 2020 Feb 21]. Available from: https://www.mscbs.gob.es/profesionales/farmacia/CIPMyPS.htm.

10. Badia X, Gil A, Poveda-Andrés JL, Shepherd J, Tort M. Analysing criteria for price and reimbursement of orphan drugs in Spain. Farm Hosp. Grupo Aula Medica S.L. 2019;43:121-7.
11. CHMP. ALPROLIX: Summary of product characteristics [Internet]. Available from: https://www.ema.europa.eu/en/documents/product-information/ alprolix-epar-product-information_en.pdf.

12. CHMP. BRONCHITOL: Summary of product characteristics [Internet]. Available from: https:/www.ema.europa.eu/en/documents/productinformation/bronchitol-epar-product-information_en.pdf.

13. CHMP. HOLOCLAR: Summary of product characteristics [Internet]. Available from: https://www.ema.europa.eu/en/documents/product-information/ holoclar-epar-product-information_en.pdf.

14. CHMP. IDELVION: Summary of product characteristics [Internet]. Available from: https://www.ema.europa.eu/en/documents/product-information/ idelvion-epar-product-information_en.pdf.

15. CHMP. NEXOBRID: Summary of product characteristics [Internet]. Available from: https://www.ema.europa.eu/en/documents/product-information/ nexobrid-epar-product-information_en.pdf.

16. CHMP. PROCYSBI: Summary of product characteristics [Internet]. Available from: https://www.ema.europa.eu/en/documents/product-information/ procysbi-epar-product-information_en.pdf.

17. CHMP. TOBI PODHALER: Summary of product characteristics [Internet]. Available from: https://www.ema.europa.eu/en/documents/productinformation/tobi-podhaler-epar-product-information en.pdf.

18. CHMP. XERMELO: Summary of product characteristics [Internet]. Available from: https://www.ema.europa.eu/en/documents/product-information/ xermelo-epar-product-information_en.pdf.

19. CHMP. KYMRIAH: Summary of product characteristics [Internet]. Available from: https://www.ema.europa.eu/en/documents/product-information/ kymriah-epar-product-information_en.pdf.

20. CHMP. REVESTIVE: Summary of product characteristics [Internet]. Available from: https://www.ema.europa.eu/en/documents/product-information/ revestive-epar-product-information_en.pdf.

21. AELMHU.es [Internet]. AELMHU Present. los datos acceso a los Medicam. huérfanos en España. [cited 2020 Feb 21]. Available from: https://aelmhu.es/ index.php/actividades/eventos-aelmhu/item/909-aelmhu-presenta-los-datosde-acceso-a-los-medicamentos-huerfanos-en-espana.

22. Paulden M, Stafinski T, Menon D, McCabe C. Value-Based Reimbursement Decisions for Orphan Drugs: A Scoping Review and Decision Framework. Pharmacoeconomics: Springer International Publishing; 2015. p. 255-69.

23. Annemans L, Aymé S, Le Cam Y, Facey K, Gunther P, Nicod E, et al. Recommendations from the European working Group for Value Assessment and Funding Processes in rare diseases (ORPH-VAL) [internet]. Orphanet ] Rare Dis Biomed Central Ltd. 2017:50 [cited 2020 Feb 21]Available from: http://www.ncbi.nlm.nih.gov/pubmed/28283046.

24. Gilabert-Perramon A, Torrent-Farnell J, Catalan A, Prat A, Fontanet M, PuigPeiró $R$, et al. Drug evaluation and decision making in catalonia: development and validation of a methodological framework based on multi-criteria decision analysis (MCDA) for orphan drugs. Int J Technol Assess Health Care. 2017:33:111-20.

25. Goetghebeur MM, Wagner M, Khoury H, Levitt RJ, Erickson LJ, Rindress D. Bridging health technology assessment (HTA) and efficient health care decision making with multicriteria decision analysis (MCDA): Applying the evidem framework to medicines appraisal. Med Decis Mak [Internet]. 2012 [cited 2020 Feb 21];32:376-88. Available from: http://www.ncbi.nlm.nih.gov/ pubmed/21987539.

26. Ministerio de Sanidad, Consumo y Bienestar Social - Profesionales de la Salud - Buscador situación financiación medicamentos [Internet]. [cited 2020 Jan 28]. Available from: https:/www.mscbs.gob.es/profesionales/ medicamentos.do.

27. European Commission: Community Register of orphan medicinal products [Internet]. [cited 2020 Jan 27]. Available from: https://ec.europa.eu/health/ documents/community-register/html/reg_od_act.htm?sort=a.

28. European Medicines Agency | [Internet]. [cited 2020 Jan 27]. Available from: https://www.ema.europa.eu/en

29. CIMA: Centro de información de medicamentos [Medicine Online Information Centre] [Internet]. [cited 2020 Jan 28]. Available from: http:// cima.aemps.es/cima/publico/home.html.

30. BOE. Ley $29 / 2006$, de 26 de julio, de garantías y uso racional de los medicamentos y productos sanitarios. BOE núm. 178, de 27 Julio de 2006 p. 28122-165. [Internet]. [cited 2020 Jan 29]. Available from: https://www.boe. es/buscar/doc.php?id=BOE-A-2006-13554

31. BOE. Real Decreto-ley $9 / 2011$, de 19 de agosto, de medidas para la mejora de la calidad y cohesión del sistema nacional de salud, de contribución a la 
consolidación fiscal, y de elevación del importe máximo de los avales del Estado para 2011. BOE núm. 200, de 20 de Agosto de 2011 p. 93143-168. [Internet]. [cited 2020 Jan 29]. Available from: https:/www.boe.es/buscar/ act.php?id=BOE-A-2011-14021\&p=20131030\&tn=2.

32. BOE. Real Decreto Legislativo 1/2015, de 24 de julio, por el que se aprueba el texto refundido de la Ley de garantías y uso racional de los medicamentos y productos sanitarios. BOE núm. 177, de 25 de julio de 2015, páginas 62935 a 63030. [Internet]. [cited 2020 Jan 29]. Available from: https://www.boe.es/ buscar/act.php?id=BOE-A-2015-8343\&p=20150725\&tn=2.

33. AETS - Instituto de Salud Carlos III M de E y C. Criterios de financiación y reembolso de los medicamentos huérfanos [Internet]. 2016. Available from: http://publicaciones.isciii.es.

34. Powers JH, Patrick DL, Walton MK, Marquis P, Cano S, Hobart J, et al. Clinician-reported outcome assessments of treatment benefit: report of the ISPOR clinical outcome assessment emerging good practices task force. Value Heal Elsevier Ltd. 2017;20:2-14.

35. AEMPS: Informes de posicionamiento terapéutico [Therapeutic positioning report] [Internet]. [cited 2020 Jan 29]. Available from: https://www.aemps. gob.es/medicamentos-de-uso-humano/informes-de-posicionamientoterapeutico/.

36. Sperandei S. Understanding logistic regression analysis. Biochem Medica Croatian Society for Medical Biochemistry and Laboratory Medicine. 2014;24: $12-8$

\section{Publisher's Note}

Springer Nature remains neutral with regard to jurisdictional claims in published maps and institutional affiliations.

Ready to submit your research? Choose BMC and benefit from:

- fast, convenient online submission

- thorough peer review by experienced researchers in your field

- rapid publication on acceptance

- support for research data, including large and complex data types

- gold Open Access which fosters wider collaboration and increased citations

- maximum visibility for your research: over $100 \mathrm{M}$ website views per year

At BMC, research is always in progress.

Learn more biomedcentral.com/submissions 